\title{
Modeling of spin-dependent hot-electron transport in the spin-valve transistor
}

\author{
R. Vlutters, O. M. J. van 't Erve, R. Jansen, S. D. Kim, and J. C. Lodder \\ Systems and Materials for Information storage, $\mathrm{MESA}^{+}$Research Institute, University of Twente, 7500 AE, Enschede, The Netherlands \\ A. Vedyayev \\ Department of Physics, Moscow Lomonosov University, Moscow 119699, Russia \\ B. Dieny \\ CEA, Département de Recherche Fondamentale sur la Matière Condensée, SP2M/NM, 38054 Grenoble Cedex 9, France
}

(Received 19 January 2001; revised manuscript received 22 May 2001; published 17 December 2001)

\begin{abstract}
A phenomenological model is presented that describes spin-dependent hot-electron transport in the spinvalve transistor. The three-dimensional model is based on the Boltzmann equation and takes into account spin-dependent inelastic and elastic scattering within each metal layer of the base and elastic scattering at the interfaces, as well as the injection and collection characteristics of the emitter and collector Schottky barriers. We numerically calculate the attenuation of the hot electrons, as well as their angular distribution of momentum, as a function of the position in the metallic base. We investigate how elastic scattering affects the attenuation lengths via the angular distribution of momentum and show that elastic scattering at an interface leads to an increase of the effective bulk attenuation length of the layers after that interface. We also find that the magnetocurrent is changed by interface scattering even if it is taken to be independent of spin. We find that when elastic scattering is significant, the true attenuation lengths are markedly larger than those predicted in a one-directional model from the scattering parameters for elastic and inelastic scattering. The calculations demonstrate that elastic scattering may be the primary reason for the small collector currents observed in the spin-valve transistor experimentally.
\end{abstract}

DOI: 10.1103/PhysRevB.65.024416

PACS number(s): 75.70.Pa, 72.10.-d, 73.40.Vz, 73.23.Ad

\section{INTRODUCTION}

Since the discovery of giant magnetoresistance, many new spin-electronic devices have been proposed. The spinvalve transistor ${ }^{1,2}$ (SVT) was introduced in 1995 as a promising hybrid device in which metallic magnetic multilayers were combined with silicon semiconductor technology. Huge magnetocurrent (up to 300\%) and low magnetic switching fields at room temperature have recently been reported. ${ }^{3,4}$ This makes the SVT a potential candidate for magnetic field sensor and memory applications, provided the relatively low collector current can be increased. Since it is hard to understand the low collector currents based on only the inelastic attenuation lengths of the hot electrons, it appears essential to include also elastic scattering in a theoretical description of the device. Recently, Yamauchi and Mizushima included elastic scattering in a model for a spin-valve transistor with a tunnel injector, ${ }^{5,6}$ focusing on the hot-electron energy dependence of the base transport. However, a one-directional (1d) model was used and a correct treatment of elastic scattering cannot be made. This requires a full three-dimensional (3D) model, in which elastic outscattering as well as inscattering is included, the evolution of the angular distribution of momentum of the hot electrons through the transistor base is evaluated, and the emission and acceptance characteristics of emitter and collector can properly be described. Furthermore, elastic scattering at the interfaces in the base is of importance.

Here we present such a 3D model for the transport in the SVT, phenomenologically including inelastic as well as elastic scattering. We investigate how elastic scattering affects the attenuation lengths via the angular distribution of momentum and show that elastic scattering at an interface leads to an increase of the effective bulk attenuation length of the layers after that interface. We also find that the magnetocurrent is changed by interface scattering even if it is taken to be independent of spin. A comparison between the full 3D calculation and a one-directional description is also made, showing that when elastic scattering is significant, the true attenuation lengths are markedly larger than those predicted in the one-directional model from the scattering parameters for elastic and inelastic scattering. The calculations show that elastic scattering may be the primary reason for the small collector currents obtained in the SVT experimentally.

We consider a magnetic metal base transistor, ${ }^{7,8}$ consisting of a semiconductor emitter and collector and a metallic spin-valve base. An emitter current $\left(I_{e}\right)$ is established such that nonequilibrium, hot electrons are injected from the silicon emitter into the base. The electrons can scatter in the base and only electrons with the right momentum and sufficient energy are collected, resulting in the collector current $\left(I_{c}\right)$. Due to the use of a spin valve in the base, the scattering can be controlled magnetically. In the parallel magnetic state of the spin valve, there is relatively little scattering and the collector current $\left(I_{c}^{P}\right)$ is highest. In the antiparallel magnetic state there is more scattering, which results in a collector current $\left(I_{c}^{A P}\right)$ that is lower than for the parallel state. The relative change in collector current is called the magnetocurrent (MC), which is defined analogous to the magnetoresistance as 


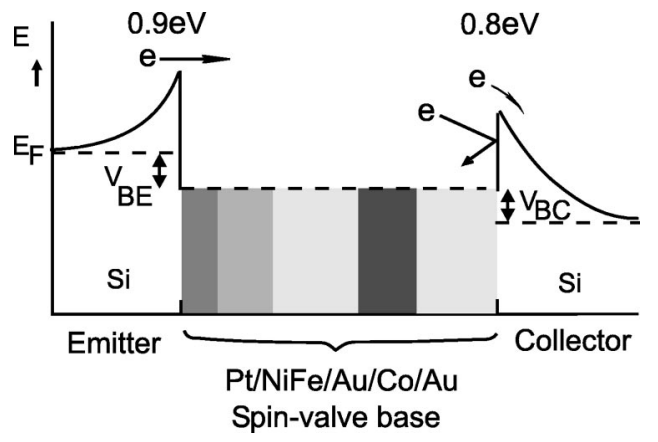

FIG. 1. The schematic energy diagram of the SVT. The electrons are injected from the Si-Pt Schottky diode with a barrier height of $0.9 \mathrm{eV}$. Electrons are collected after passing the $\mathrm{Au}-\mathrm{Si}$ Schottky barrier $(0.8 \mathrm{eV})$ on the collector side.

$$
\mathrm{MC} \equiv \frac{I_{c}^{P}-I_{c}^{A P}}{I_{c}^{A P}} .
$$

Furthermore, we defined the current transfer ratio ( $\equiv I_{c} / I_{e}$ ), which describes the proportionality between the collector and emitter current.

Due to the combination of semiconductors and metals, two Schottky barriers are formed, as shown in the schematic energy diagram (Fig. 1). Electrons are injected into the base after passing the Si/Pt Schottky barrier, which results in hot electrons with an excess energy of about $0.9 \mathrm{eV}$ above the metal Fermi level. The collector (Si/Au) Schottky barrier accepts only electrons that have enough energy to overcome the $0.8 \mathrm{eV}$ collector barrier, by that filtering out electrons that have lost their energy while traveling from the emitter to the collector. Hot electrons can lose their excess energy by inelastic scattering with the electrons from the Fermi sea, 9,10 creating electron-hole pairs. Inelastic scattering generally results in a reduction of the electron's energy to below the collector barrier, after which the electron can no longer contribute to the collector current.

Besides the energy of the hot electron, also its $k$ vector plays a role. The electrons are injected within a very narrow emission cone, as indicated in the schematic cross section of the SVT (Fig. 2). This forward focusing is due to the acceleration in the metal/semiconductor interface region, in the $x$ direction perpendicular to the interface. For the same reason, the collector only accepts electrons within a certain acceptance cone. Electrons are reflected back into the base if their direction of motion ( $k$ vector) is not within the acceptance cone. Note that in principle one has to take the full band structure of the semiconductor and metals into account, which is beyond the scope of the present work. We here use the concept of a collector cone, which is calculated assuming a free-electron model with isotropic effective masses. Taking the $k$ vector into account makes the collector current also dependent on elastic scattering mechanisms in which only the momentum, but not the energy of the electron changes. Possible sources are defects, impurities, stacking faults, or mismatches of the band structure. Thus, elastic scattering can remove an electron from the acceptance cone (see path No. 3 in Fig. 2), but it can also scatter an electron that was already

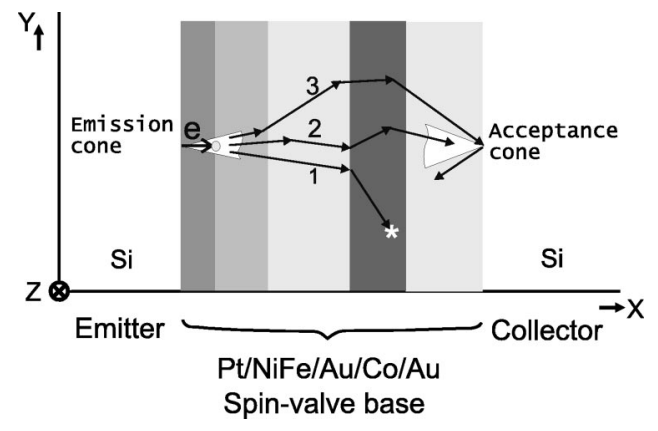

FIG. 2. A schematic cross section of the SVT. Three possible paths of an electron traveling through the base of the SVT are drawn: (1) an electron scatters inelastically (as indicated by the asterisk) after which it is lost, (2) an electron scatters elastically several times, but still arrives within the acceptance cone of the collector, or (3) an electron scatters elastically and arrives at the collector outside the acceptance cone, and thus will be reflected back into the base.

outside the acceptance cone back into it (path 2 in Fig. 2). Both possibilities are taken into account in our model.

In order to model the electron transport in the SVT, we have to calculate the collector current taking into account the spin dependence of the elastic and inelastic scattering events in the ferromagnetic layers. The electron transport in the SVT is calculated separately for spin-up and spin-down electrons, because no spin mixing is included, after which the spin currents are added to give the total electron current.

One of the main differences in the following current transport calculations in the SVT, as compared to giant magnetoresistance in metallic multilayers, ${ }^{11-14}$ is that there is no electrical field in the base region of the SVT to drive the electrons from the emitter to the collector. Electrons have to use their kinetic energy to travel through the base and arrive in the collector. This results in a much stronger dependence of main characteristic values (such as the magnetocurrent) on the thickness of the layers.

In the next section we explain how to use the Boltzmann equation to describe the hot-electron transport through the base while incorporating elastic and inelastic scattering events, and calculate the specific boundary conditions due to the Schottky barriers at the emitter and collector side. After solving these equations using a procedure described in the Appendix A, the numerical results are presented in Sec. III. We show the main characteristics of the current transport and explain how the spin-dependent scattering in the magnetic layers of the base results in a high magnetocurrent. Furthermore, we discuss the dependence of the collector current on the thickness of the magnetic layers and the degree of elastic interface scattering.

\section{THEORY}

\section{A. Boltzmann equation}

In order to model the electron transport we use the semiclassical Boltzmann equation within the metal base region. The influence of the emitter and collector Schottky barriers are included in the boundary conditions. This approach gives 
the flexibility to incorporate various physical scattering mechanisms and gives good insight into their effect on the transport. We start from the general Boltzmann equation ${ }^{15}$

$$
\begin{array}{r}
\frac{\partial f(\vec{x}, \vec{v}, t)}{\partial t}+\frac{\partial f(\vec{x}, \vec{v}, t)}{\partial \vec{x}} \cdot \frac{\partial \vec{x}}{\partial t}+\frac{\partial f(\vec{x}, \vec{v}, t)}{\partial \vec{v}} \cdot \frac{\partial \vec{v}}{\partial t} \\
=-\sum_{v^{\prime}}\left[W_{v v^{\prime}} f(\vec{x}, \vec{v}, t)-W_{v^{\prime} v} f\left(\vec{x}, \vec{v}^{\prime}, t\right)\right],
\end{array}
$$

in which $f(\vec{x}, \vec{v}, t)$ is the complete Boltzmann distribution function describing the number of hot electrons in a certain phase space with position $\vec{x}$ and velocity $\vec{v}$ at time $t, W_{v v^{\prime}}$ the transition rate from velocity $v$ to $v^{\prime}$, and $W_{v^{\prime} v}$ vice versa.

We can make several simplifications based on the type of current transport for hot-electrons in the base. First, we seek a stationary solution and so the first term in Eq. (2) can be ignored. Second, the electrons need to travel through the base without the help of an electrical field; i.e., there is no acceleration $(\partial \vec{v} / \partial t=0)$, and so the third term can be ignored. After these simplifications Eq. (2) reduces to

$$
\frac{\partial f(\vec{x}, \vec{v})}{\partial \vec{x}} \cdot \vec{v}=-\sum_{v^{\prime}}\left[W_{v v^{\prime}} f(\vec{x}, \vec{v})-W_{v^{\prime} v} f\left(\vec{x}, \vec{v}^{\prime}\right)\right] .
$$

By using Mathiesen's rule ${ }^{15}\left(W_{v v^{\prime}}=\Sigma_{s} W_{v v^{\prime}}^{s}\right)$ we can now include all scattering mechanisms, each with their own scattering rate. In this paper we assume isotropic elastic and inelastic scattering, without using the relaxation time approximation.

Let us define the exact nature of the scattering mechanisms as included in the model. Inelastic scattering $\left(W^{i}\right)$ is modeled as a process which lowers the energy of an electron to below the collector barrier, resulting in an effective loss for the collector current. This gives only outscattering $\left(W_{v v^{\prime}}\right)$, and thus

$$
-\sum_{v^{\prime}}\left[W_{v v^{\prime}}^{i} f(\vec{x}, \vec{v})-W_{v^{\prime} v}^{i} f\left(\vec{x}, \vec{v}^{\prime}\right)\right]=-\frac{f(\vec{x}, \vec{v})}{\tau_{i}} .
$$

It should be noted that this makes the inelastic lifetime $\left(\tau_{i}\right)$, as relevant for the SVT, dependent on the maximum allowed energy loss and thereby on the difference between emitter and collector Schottky barrier height. In general, inelastic scattering by electron-hole pair creation involves large energy loss. However, for a large Schottky barrier difference, part of the inelastic scattering events may not lower the electron energy to below the collector barrier maximum.

Elastic scattering $\left(W^{e}\right)$ can remove (outscattering) an electron from the state with velocity/direction $v$ with time constant $\tau_{e}$, but can also scatter an electron from the state with velocity $v^{\prime}$ into one with velocity $v$ (inscattering), where detailed balancing requires $W_{v^{\prime} v}^{e}=W_{v v^{\prime}}^{e}$. For the elastic scattering we thus have

$$
\begin{aligned}
& -\sum_{v^{\prime}}\left[W_{v v^{\prime}}^{e} f(\vec{x}, \vec{v})-W_{v^{\prime} v}^{e} f\left(\vec{x}, \vec{v}^{\prime}\right)\right] \\
& =-\frac{f(\vec{x}, \vec{v})}{\tau_{e}}+\frac{1}{4 \pi|v|^{2} \tau_{e}} \int f\left(\vec{x}, \vec{v}^{\prime}\right) \delta\left(|\vec{v}|-\left|\vec{v}^{\prime}\right|\right) d \vec{v}^{\prime},
\end{aligned}
$$

where the first term on the right-hand side describes the outscattering and the second term the inscattering. Thus, no hot electrons are lost in an elastic scattering event; only the momentum direction is changed. Elastic scattering in our model includes all scattering events after which the hot electron still has enough energy to overcome the collector barrier; that is, quasielastic scattering mechanisms are included as purely elastic.

These definitions simplify the numerical calculation significantly, as one does not have to calculate the evolution of the energy distribution of the hot electrons. After including the elastic and inelastic scattering mechanisms in Eq. (3) we obtain

$$
\begin{aligned}
\frac{\partial f(\vec{x}, \vec{v})}{\partial \vec{x}} \cdot \vec{v}= & -\left[\frac{1}{\tau_{e}}+\frac{1}{\tau_{i}}\right] f(\vec{x}, \vec{v})+\frac{1}{4 \pi|v|^{2} \tau_{e}} \\
& \times \int f\left(\vec{x}, \vec{v}^{\prime}\right) \delta\left(|\vec{v}|-\left|\vec{v}^{\prime}\right|\right) d \vec{v}^{\prime} .
\end{aligned}
$$

In order to reduce the number of calculations and to facilitate the integral calculation we will assume that all the hot electrons have the same magnitude of velocity. Using this simplification we can convert the lifetimes into mean free paths $(\lambda=v \tau)$ and transform the integral in Eq. (6) to an integration over the surface of a sphere. Making use of the cylindrical symmetry of the SVT (equivalence of the $y$ and $z$ axes; see Fig. 2), we obtain

$$
\mu \frac{\partial f(x, \mu)}{\partial x}=-\left[\frac{1}{\lambda_{e}}+\frac{1}{\lambda_{i}}\right] f(x, \mu)+\frac{1}{2 \lambda_{e}} \int_{-1}^{1} f(x, \mu) d \mu .
$$

Here, $f(x, \mu)$ is the Boltzmann distribution function describing the number of electrons per second at position $x$ with momentum in the direction $\mu$, where $\mu$ is defined as the cosine of $\theta$, the solid angle between the velocity vector and the $x$ axis of the transistor. After numerically solving Eq. (7), which is explained in Appendix A, we can calculate the current in the positive $x$ direction from the distribution function using

$$
I_{x}^{>}(x)=q \int_{0}^{1} \mu f(x, \mu) d \mu,
$$

where $q$ is the electron charge.

Equations (7) and (8) form the basis of our model calculations. However, it is instructive to compare with a purely one-directional model in which only electrons with their momentum exactly in the positive $x$ direction $(\mu=1)$ are considered. In this case, any elastic or inelastic scattering event definitively removes an electron from the collector current 


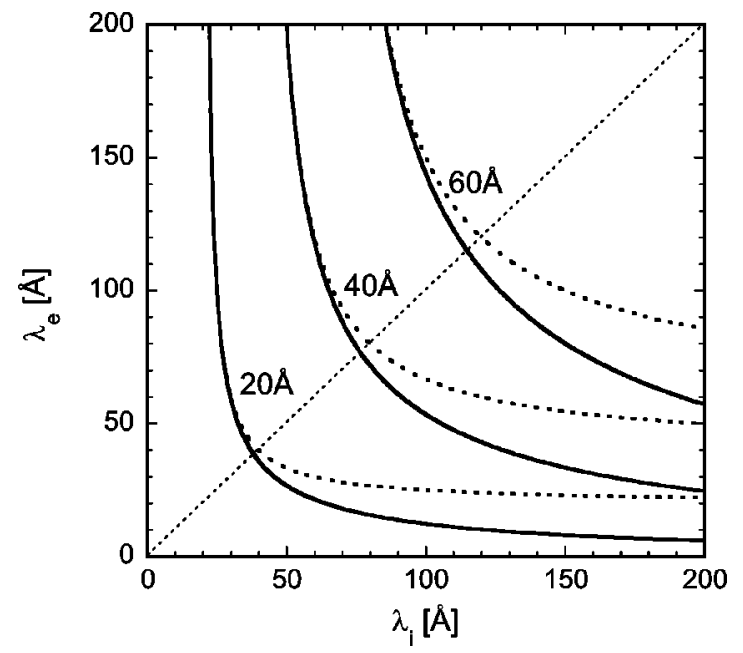

FIG. 3. The different combinations of $\lambda_{e}$ and $\lambda_{i}$ that result in the same $\lambda_{e f f}=20,40$, and $60 \AA$. The solid lines denote the 3D model; the dashed lines refer to the one-directional model.

and no correct treatment of elastic inscattering can be made. Equation (7) can be written in this one-directional approach as

$$
\frac{\partial f(x)}{\partial x}=-\left[\frac{1}{\lambda_{e}}+\frac{1}{\lambda_{i}}\right] f(x) .
$$

This can easily be solved, resulting in

$$
f(x)=f_{0} \exp \left(-\frac{x}{\lambda_{e f f}}\right) .
$$

Here, $f_{0}$ is the distribution at the emitter-base interface ( $x$ $=0)$ and $f(x)$ is the position-dependent distribution function that decays exponentially with the characteristic length $\lambda_{e f f}$, as does the current. In this case, $\lambda_{e f f}$ is simply $\left(\lambda_{e}^{-1}\right.$ $\left.+\lambda_{i}^{-1}\right)^{-1}$.

In one specific case it is possible to solve Eq. (7) analytically, if one assumes a purely exponential attenuation (see the Appendix). This results in a direct relation between $\left(\lambda_{e}, \lambda_{i}\right)$ and $\lambda_{e f f}$. In Fig. 3, different combinations of $\lambda_{e}$ and $\lambda_{i}$ are shown that result in the same $\lambda_{e f f}$ as given by the solid line. The result for the one-directional model is also shown (dashed line) and deviates significantly from the analytical solution when the elastic scattering length is much shorter than the inelastic scattering length. For example, when $\lambda_{i}=200 \AA$ and $\lambda_{e}=25 \AA$ the distribution function decays exponentially in the one-directional model with a characteristic length of $\approx 22 \AA$, but the analytical 3D solution gives $40 \AA$, almost twice as long. This is because electrons that are elastically scattered to directions outside the acceptance cone, can still scatter back into the acceptance cone within the 3D model. This clearly demonstrates that in the case of strong elastic scattering, the elastic inscattering should be taken into account and no one-directional approximation can be made. The analytical solution correctly takes the full elastic inscattering into account, but can only be applied in a uniform base without boundary conditions. Therefore, a numerical method based on Eq. (7) is used, as described in the Appendix A, to solve the Boltzmann equation inside the spin-valve transistor.

Knowing how to calculate the hot-electron flow through a single layer, we focus in this paragraph on how to implement the multilayer base, as found in the SVT. First one has to take inside every layer the corresponding bulk scattering parameters $(\lambda$ 's). Next one has to include interface scattering and relate the solutions to the Boltzman equation at both sides of the interface to each other. In this article, elastic interface scattering that occurs at the metal-metal as well as at the silicon-metal interfaces is introduced with the transport parameter $D$, which represents the interface diffusivity. From the incident distribution of electrons, a fraction $(1-D)$ crosses the interface without scattering, while a fraction $D$ scatters at the interface. The elastic interface scattering is assumed to be isotropic, such that a fraction $D / 2$ of the electrons still moves in the original direction, but the other $D / 2$ is scattered into the opposite direction. Physically, this elastic interface scattering is related to the defects, impurities, and stacking faults at the interfaces, as well as to the abrupt change in electrical properties (band structure).

Essential in the calculation of the current through the base of the SVT is to take into account that in ferromagnetic materials the mean free path is spin dependent. When a spin-up electron travels through a ferromagnet whose magnetization is aligned with the electron's spin, it is weakly scattered (with longer $\lambda_{e}^{\uparrow}$ and $\lambda_{i}^{\uparrow}$ ), while an electron with spin opposite to the magnetization scatters more strongly (shorter $\lambda_{e}^{\downarrow}$ and $\left.\lambda_{i}^{\downarrow}\right)$. Note that in principle also the interface scattering can be spin dependent. However, in this paper we only include spin-independent interface scattering to show the effect of it on the current transfer ratio. In the presented calculations, we start with 50\% spin-up electrons and 50\% spindown electrons, as injected from the nonmagnetic emitter. We then calculate for both spin channels the resulting collector current and add them to obtain the total collector current. This can be done for the parallel and antiparallel configurations of the two magnetic layers and results finally in the MC. In the developed model, no spin-flip scattering was taken into account, so that the current of spin-up electrons can be calulated independent from the spin-down current.

\section{B. Boundary conditions: Schottky diodes}

The electrons in the spin-valve transistor are injected from a Schottky diode and are also collected by a Schottky diode on the collector side. Schottky diodes are known to inject and collect in a very narrow angle (typically less than $10^{\circ}$ ), which is why a detailed analysis of the angular distribution of momentum during transport through the base is important.

Let us first calculate the angular distribution of the emitter current $\left(I_{e}\right)$. We start with the thermionic emission equation for electron transport over a Schottky barrier ${ }^{16}$ :

$$
\begin{aligned}
I= & A \cdot A^{* *} T^{2} e^{-q V_{n} / k T} \int_{\sqrt{\left(2 q / m_{s i}\right)\left(V_{b i}-V\right)}}^{\infty} d v_{s x} \cdot v_{s x} e^{-m_{s i} v_{s x}^{2} / 2 k T} \\
& \times\left[\int_{-\infty}^{\infty} \int_{-\infty}^{\infty} d v_{s y} \cdot d v_{s z} e^{-m_{s i}\left(v_{s y}^{2}+v_{s z}^{2}\right) / 2 k T}\right]
\end{aligned}
$$


This is essentially an integral over the electron distribution inside the semiconductor, with $v_{s x}, v_{s y}, v_{s z}$ the velocity of the electrons and $m_{s i}$ the effective mass in the (silicon) semiconductor. $V_{n}$ is the difference between the Fermi level and the conduction-band minimum and $V_{b i}$ is the so-called "built-in potential" due to the band bending in the depletion region. Furthermore, $V$ is the applied voltage, $A$ the area of the diode, and $A^{* *}$ the effective Richardson constant. Since we need the angular distribution in the metal base, we have to rewrite the integral in terms of the electron velocities in the metal (with $v_{m x}, v_{m y}, v_{m z}$ ). These are different from those in the semiconductor due to the lower conduction-band minimum and the higher effective mass in the metal. Using energy conservation in the $x$ direction and parallel momentum conservation in the $y / z$ direction, we obtain

$$
\begin{gathered}
v_{s x} \cong \sqrt{\frac{m}{m_{s i}} v_{m x}^{2}-\frac{2 q}{m_{s i}}\left(\Phi_{b}+E_{f m}+V-V_{b i}\right)}, \\
v_{s y}=\left(\frac{m}{m_{s i}}\right) v_{m y}, \quad v_{s z}=\left(\frac{m}{m_{s i}}\right) v_{m z},
\end{gathered}
$$

where $\Phi_{b}$ is the Schottky barrier height and $E_{f m}$ the difference between the conduction-band minimum and the Fermi level in the metal. Transforming Eq. (11) using these relations results in exactly the same current but expressed in the metal system coordinates. In order to obtain the boundary condition for the base transport equation, we have to express this integral in the same spherical coordinates as Eq. (7), so the transformations are

$$
\begin{gathered}
v_{m x}=v_{a b s} \mu, \quad v_{m y}=v_{a b s} \sqrt{1-\mu^{2}} \cos (\phi), \\
v_{m z}=v_{a b s} \sqrt{1-\mu^{2}} \sin (\phi),
\end{gathered}
$$

in which $v_{a b s}$ is the absolute velocity in the metal, $\mu$ the directional cosine, and $\phi$ the angle of the projection on the $y-z$ plane with the y axis. After an integration over only $v_{a b s}$ and $\phi$, the $\mu$-resolved current $I(\mu)$ is obtained. The angular distribution function on the emitter side can now be obtained from $f(x=0, \mu)=I(\mu) /(q \mu)$. In Fig. 4, we plot the angular emission of a Schottky diode with $\Phi_{b}=0.9 \mathrm{eV}$, showing clearly the forward-focused character of the injected electrons. It should be noted that increasing the voltage over the emitter-base contact only changes the amount of electrons that is injected into the base, but it does not change their energy and momentum distribution.

Having described the angular emission spectrum of the emitter Schottky barrier, we now consider the collector transmission spectrum. In order to calculate the angular collection characteristics of a Schottky diode we assumed an abrupt step in conduction-band minimum between the metallic base and the silicon collector. This results in a quantum mechanical reflection and transmission ${ }^{17,18}$ which is a function of the incoming and transmitted velocity perpendicular to the interface $\left(v_{i}^{\perp}\right.$ and $v_{t}^{\perp}$, respectively):

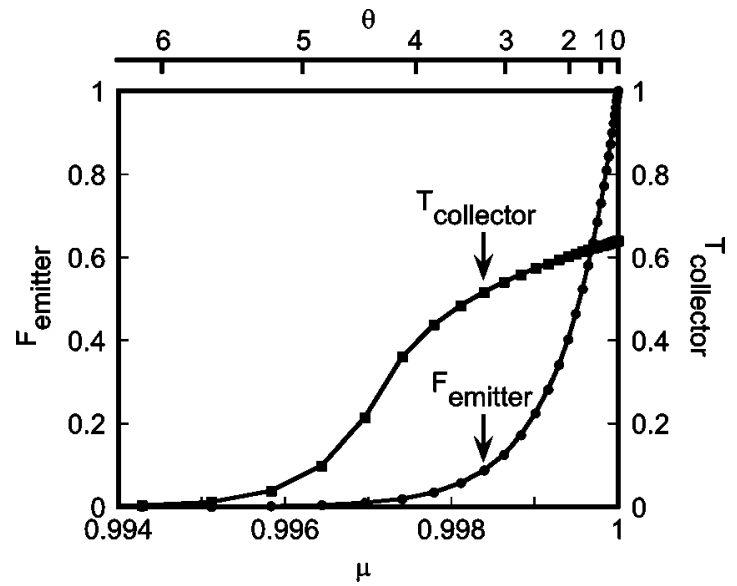

FIG. 4. The emission spectrum for the emitter $\left(F_{\text {emitter }}\right)$ and the transmission spectrum for the collector $\left(T_{\text {collector }}\right)$ as function of $\mu=\cos \theta$. The electrons are emitted over an $0.9 \mathrm{eV}$ Schottky barrier and collected in an $0.8 \mathrm{eV}$ Schottky diode at room temperature and $E_{F M}=5.5 \mathrm{eV}$ for both barriers. The emission spectrum has been normalized to 1 for electrons with their momentum parallel to the $x$ axis (the dots and squares are the discretization points of $\mu$ ).

$$
R=\left|\frac{v_{i}^{\perp}-v_{t}^{\perp}}{v_{i}^{\perp}+v_{t}^{\perp}}\right|^{2}, \quad T=1-R .
$$

Using an equation similar to Eq. (12) to calculate $v_{t}^{\perp}$ as a function of the incoming velocity, and applying a transformation like Eq. (13), we obtain the transmission as a function of $v_{a b s}$ and $\mu$. Because the transmission is dependent on the velocity $\left(v_{a b s}\right)$ of the incoming electrons, we use a velocity-resolved emission spectrum from the emitter. So we sample the transmission function with the normalized $v_{a b s}$-resolved emitter current and this finally results in the angular dependence of the collection factor:

$$
T_{\text {collector }}(\mu)=\int T_{\text {collector }}\left(v_{\text {abs }}, \mu\right) I_{\text {emitter }}^{\text {normalized }}\left(v_{a b s}\right) d v_{a b s} .
$$

This function is plotted in Fig. 4 for a $0.8 \mathrm{eV}$ collector barrier when the electrons are emitted from a $0.9 \mathrm{eV}$ emitter barrier. We see that the collection spectrum is slightly broader than the emission spectrum. This ensures the collection of electrons that are not elastically scattered, but causes reflection of most of the elastically scattered electrons.

\section{NUMERICAL RESULTS}

The spin-valve transistor that we consider here has the same structure as used in our latest experiments. It consists of two Si wafers with the following base: Pt $(20 \AA) / \mathrm{NiFe}(30$ $\AA) / \mathrm{Au}(35 \AA) / \mathrm{Co}(30 \AA) / \mathrm{Au}(20 \AA) / / \mathrm{Au}(20 \AA)$ (" $/ / /$ " indicates the bond interface). When injecting an emitter current of $2 \mathrm{~mA}$, a collector current of $11 \mathrm{nA}$ was measured with an MC of $200 \%$ at room temperature. ${ }^{3}$

Essential to any simulation is the use of realistic material parameters. Many of the scattering lengths at $0.9 \mathrm{eV}$ above the Fermi level are not precisely known. In a recent BEEM 
TABLE I. The materials in the base of the SVT, their thickness $(W)$, and the corresponding elastic $\left(\lambda_{e}\right)$ and inelastic $\left(\lambda_{i}\right)$ mean free paths used in the calculations. Furthermore, the effective attenuation lengths based on the one-directional and analytical threedimensional model are shown in the last two columns (all lengths are in angstroms $[\AA]$ ).

\begin{tabular}{lccccc}
\hline \hline Material & $W$ & $\lambda_{e}(\uparrow / \downarrow)$ & $\lambda_{i}(\uparrow / \downarrow)$ & $\lambda_{\text {eff }}^{1-d}(\uparrow / \downarrow)$ & $\lambda_{\text {eff }}^{3 D}(\uparrow / \downarrow)$ \\
\hline $\mathrm{Pt}$ & 20 & 50 & 100 & 33 & 39 \\
\hline $\mathrm{NiFe}$ & 30 & $30 / 10$ & $160 / 80$ & $25 / 9$ & $39 / 16$ \\
\hline $\mathrm{Au}$ & 35 & 200 & 400 & 133 & 155 \\
\hline $\mathrm{Co}$ & 30 & $30 / 10$ & $160 / 80$ & $25 / 9$ & $39 / 16$ \\
\hline $\mathrm{Au}$ & 20 & 200 & 400 & 133 & 155 \\
\hline $\mathrm{Au}$ & 20 & 200 & 400 & 133 & 155 \\
\hline \hline
\end{tabular}

study, ${ }^{19}$ the attenuation lengths for electrons at $1 \mathrm{eV}$ above the Fermi level in Co were measured $\left(\lambda_{\text {att }}^{\uparrow} \approx 23 \AA, \lambda_{\text {att }}^{\downarrow}\right.$ $\approx 8 \AA$ ). Both elastic and inelastic scattering contribute to this attenuation length. Inelastic lifetimes have been probed in a recent time- and spin-resolved two-photon photoemission experiment ${ }^{18,20}$ for Co. At about $1 \mathrm{eV}$, the measured inelastic lifetimes are $\tau_{i}^{\uparrow} \approx 8$ fs and $\tau_{i}^{\downarrow} \approx 4 \mathrm{fs}$. Based on these inelastic lifetimes and a velocity of $v \approx 20 \AA /$ fs for hot electrons in an $s$ band, we can calculate the inelastic scattering length with $\lambda_{i}=v \tau_{i}$, resulting in $\lambda_{i}^{\uparrow}=160 \AA$ and $\lambda_{i}^{\downarrow}$ $=80 \AA$. Thus, inelastic scattering alone is not sufficient to obtain total attenuation lengths of 23 and $8 \AA$, respectively. Therefore, we add elastic scattering with $\lambda_{e}^{\uparrow}=30 \AA$ and $\lambda_{e}^{\downarrow}$ $=10 \AA$. For NiFe, no experimental data were available, so we choose the same values as for Co. It should be noted that based on the used elastic and inelastic scattering lengths in $\mathrm{Co}$ and $\mathrm{NiFe}$, an electron can on the average scatter elastically $\approx 5$ times before it loses its energy in an inelastic scattering event. For gold, there are many BEEM studies, ${ }^{21,22}$ all reporting slightly different values, but in general one finds much larger scattering lengths than for Co. Furthermore, the measured values are much larger than the layer thickness, so bulk scattering in the gold layer has a small influence on the transfer ratio $\left(I_{c} / I_{e}\right)$. Finally, for the platinum layer an attenuation length on the order of $40 \AA$ was measured in a BEEM study on Pt silicide. ${ }^{23}$ Because the elastic and inelastic contributions are not known separately, we have simply chosen values in agreement with this total attenuation. All the used bulk scattering parameters are summarized in Table I and the interface diffusivity $D$ has been set to 0.9 for all interfaces, unless stated otherwise. This was found to result in a collector current similar to the experimental values. We have, however, also varied the interface diffusivity and examined the influence on the collector current. It should be noted that experimental MC values can be higher than the values calculated here, because no spin-dependent interface scattering was taken into account.

A first important characteristic of the current transport in the SVT is the exponential decay of the current with the position in the base layer. In Fig. 5 we have calculated the

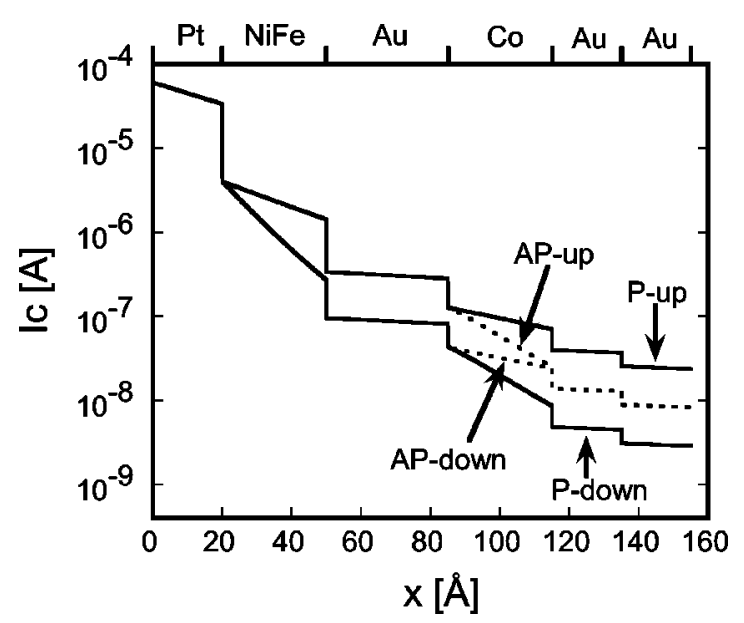

FIG. 5. The current within the collector acceptance cone as a function of the position $x$ inside the SVT for the spin-up and spindown electrons, in the parallel (P) and antiparallel (AP) magnetic states of the spin valve.

current carried by electrons with their momentum such that they are within the acceptance cone of the collector. This current is given by

$$
\begin{aligned}
I_{\text {coll }}(x) & \equiv q \int_{0}^{1} \mu T_{\text {collector }}(\mu) f(x, \mu) d \mu \\
& =q \sum_{i} a_{i} \mu_{i} T_{\text {collector }, i} f_{i}^{>}(x) .
\end{aligned}
$$

An exponential decrease of the current is obtained for both spin-up and spin-down electrons and in the parallel $(\mathrm{P})$ as well as the antiparallel (AP) magnetic state of the spin valve. Needless to say, the exponential decay varies from one layer to the next and depends on the hot-electron spin in the ferromagnetic layers. Obviously, the spin-dependent exponential decay is one of the factors that leads to the huge relative magnetic response of the SVT. This contrasts to the role of the mean free path in the giant magnetoresistance of a magnetic multilayer, where the resistances are linearly proportional to (the inverse of) the mean free path.

A further observation in Fig. 5 is that steplike current losses occur at each of the interfaces, due to elastic scattering at the interfaces having relatively strong diffusivity ( $D$ $=0.9$ ). Interface scattering at the very first Si-Pt interface results in a complete $(1-D)$ times reduction of the current inside the acceptance cone (therefore Fig. 5 starts at 6 $\times 10^{-5} \mathrm{~A}$, which is about $(1 \mathrm{~mA}) \times(1-D) \times T_{\text {collector }}(\mu$ $\approx 1$ ) for the spin-up and spin-down channels). It is, however, interesting to note that the current loss is not equal at all interfaces, even though the interface diffusivity $D$ has been set to same value of 0.9 for all interfaces. This illustrates that the effect of elastic scattering on reducing the current depends on factors other than the scattering strength. More precisely, it depends on the angular distribution of hot electrons that is incident on the scattering interface. At the $\mathrm{Pt} / \mathrm{NiFe}$ interface, the angular distribution is still strongly forward directed, and so interface scattering will result in a lot of these electrons being deflected and removed from the accep- 

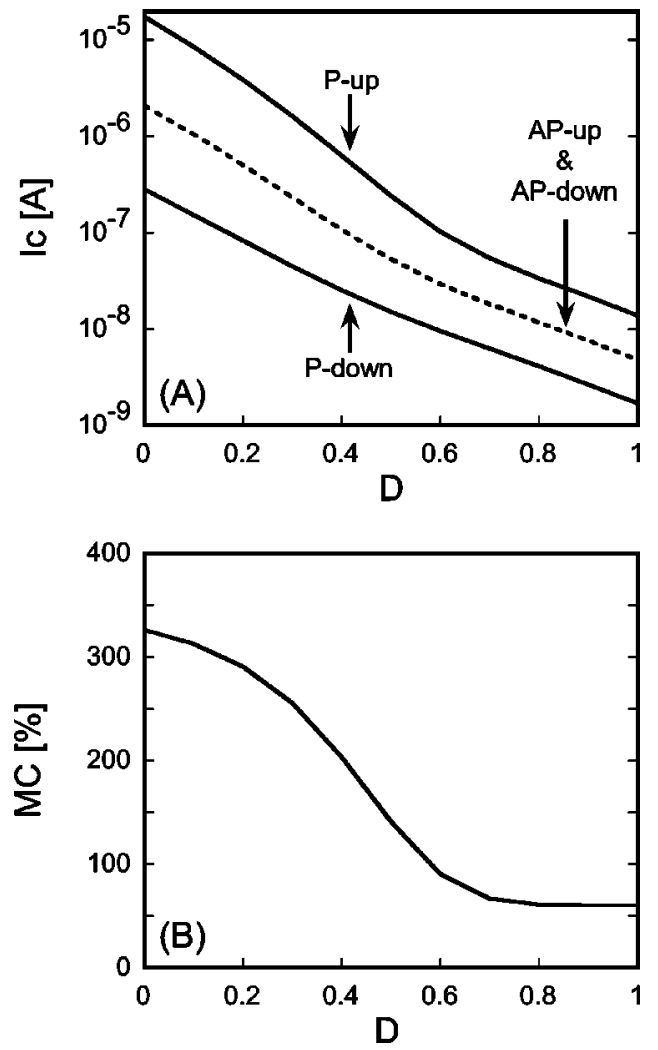

FIG. 6. The influence of elastic interface scattering $D$ on the collector current for the spin-up and -down channels in both magnetic configurations (A) and the magnetocurrent (B).

tance cone of the collector. As the hot electrons travel through the base, their angular distribution becomes more and more isotropic due to the cumulative effect of all the elastic scattering. Thus, at interfaces closer to the collector (such as the $\mathrm{Au} / \mathrm{Au}$ bond interface) the more isotropic distribution reduces the influence of additional elastic scattering. Electrons moving at large angles outside the acceptance cone, and even electrons moving in the backward direction (to the left) can now be scattered back inside the acceptance cone.

Another important point is that the current decay is found to be stronger in the NiFe layer as compared to that in the Co layer, even though the scattering parameters $\lambda_{e}$ and $\lambda_{i}$ were chosen to be exactly the same for both materials. This difference is also due to the different angular distribution at different positions in the SVT base. In the NiFe layer, which is closer to the emitter, the electron distribution is more forward directed, while in the Co layer the distribution has become more isotropic. Elastic scattering in the $\mathrm{NiFe}$ layer will thus have more effect than in the Co layer, resulting in an effective decay length that is shorter in NiFe than in Co.

In Fig. 6 we show the dramatic effect of the interface diffusivity $D$ on the collector current and the magnetocurrent. In Fig. 6(A) we see that as the interface diffusivity is increased from 0 to 1 , the collector current is reduced by about three orders of magnitude. It thus appears that strong elastic interface scattering can easily account for the low transfer ratios that are experimentally observed. Improving
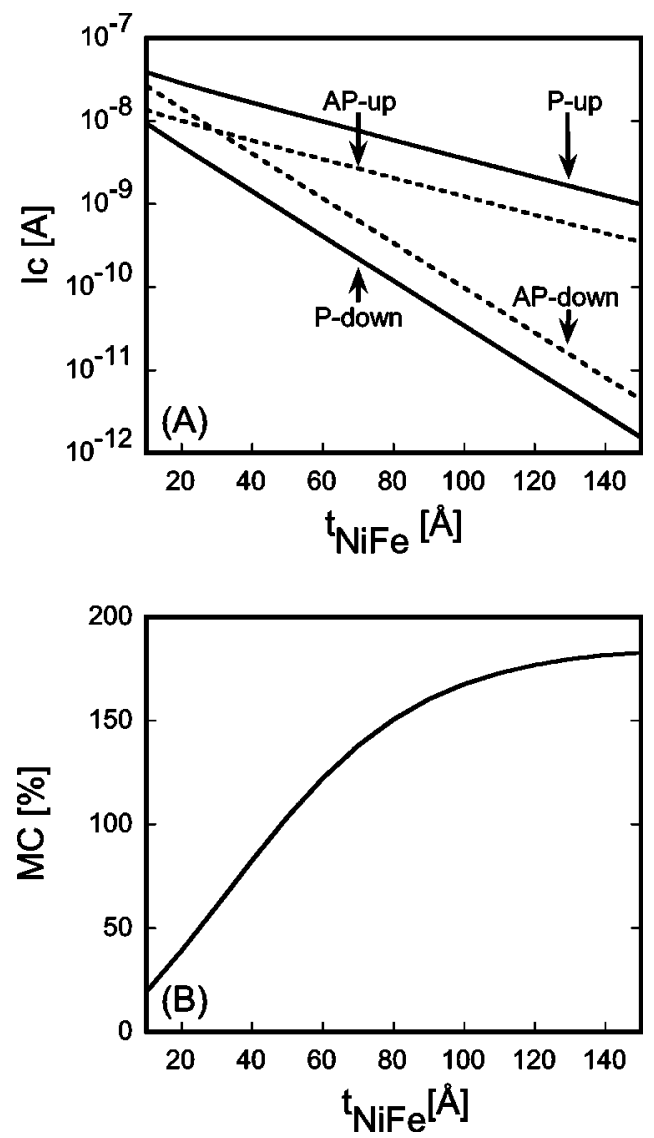

FIG. 7. The influence of the NiFe thickness on the collector current for the spin-up and-down channels in both magnetic configurations (A) and the magnetocurrent (B).

the interface quality therefore seems a promising route for increasing the output current of the SVT. The finding that interface scattering reduces the MC, as shown in Fig. 6(B), is quite remarkable since the interface scattering was taken to be independent of spin. In the simple one-directional approach it can be easily shown that a spin-independent scattering process cannot lower the MC, as it attenuates both spin currents by the same factor, leaving the ratio unchanged. Again, the explanation has to do with the influence of the angular distribution function on the current decay. For negligible diffuse interface scattering, the angular distribution remains strongly forward directed, such that the spindependent elastic bulk scattering in the magnetic layers contributes effectively to the MC. However, when diffuse interface scattering is strong, the role of bulk spin-dependent elastic scattering is diminished and therefore the MC becomes dominated by the longer inelastic bulk scattering lengths. This finally results in a lower MC. It should be noted that elastic interface scattering deflects electrons away from the film normal, thereby increasing the distance that the electrons travel through the magnetic layers. This results in an increase of the effective thickness of the magnetic layers, which tends to enhance the MC. This effect, which is included in our calculations, apparently is weaker than the angular distribution effect that is described above.

In Figs. 7 and 8 we show how the MC scales with the 

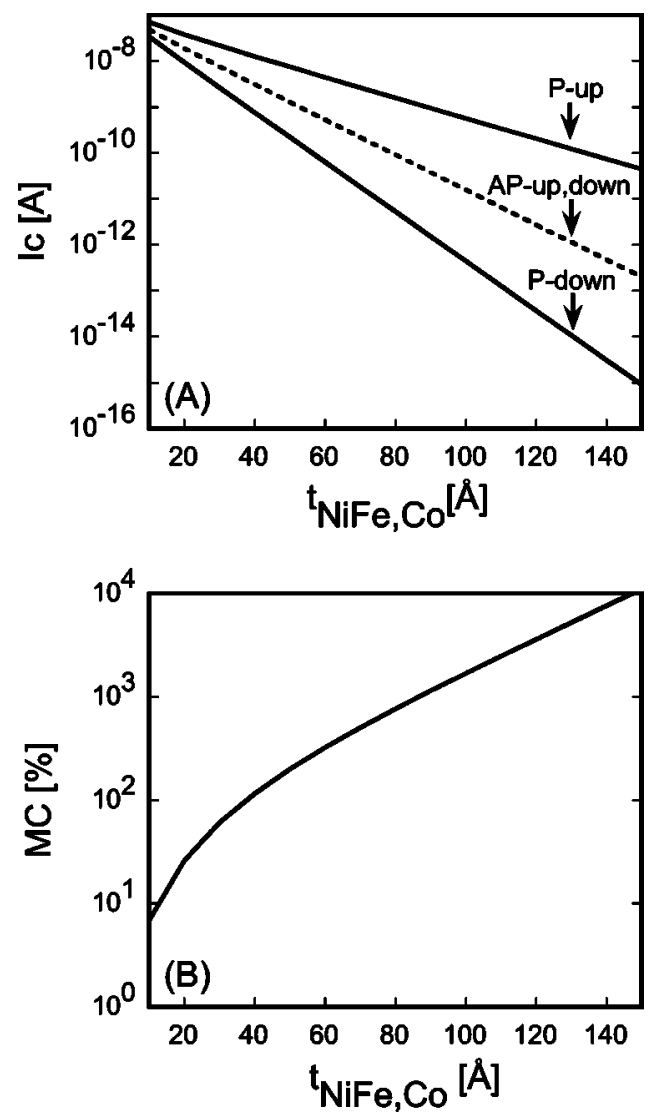

FIG. 8. The influence of the thickness of both the $\mathrm{NiFe}$ and $\mathrm{Co}$ layers on the collector current for the spin-up and -down channels in both magnetic configurations (A) and the magnetocurrent (B).

ferromagnetic layer thickness ( $\mathrm{NiFe}$ and $\mathrm{Co}$ ). First, in Fig. 7, we change only the thickness of the NiFe layer, keeping the Co layer thickness fixed at $30 \AA$. The current of electrons with spin aligned to the $\mathrm{NiFe}$ magnetization decreases exponentially with an attenuation length of $38 \AA$ as extracted from Fig. 7 and the current of electrons with their spin opposite to the NiFe magnetization attenuates with a characteristic length of $16 \AA$. Hence, for thicker NiFe layers the MC increases and it saturates at a value that is determined by the finite thickness of the fixed Co layer. This saturation occurs at a NiFe thickness of about $100 \AA$, when the NiFe transmits only majority-spin electrons. Note that the precise value of the $\mathrm{MC}$ at a given thickness may deviate from that observed experimentally when the scattering parameters $\left(\lambda_{e}, \lambda_{i}, D\right)$ differ from those used in the calculations of Figs. 7 and 8. Also recall that we have taken the interface scattering to be independent of spin.

When the observed attenuation length in NiFe is compared to the value from the one-directional model (see Table I) we see that the inclusion of elastic inscattering results in a remarkably larger attenuation length (i.e., $38 \AA$ as compared to $25 \AA$ for majority-spin electrons and $16 \AA$ as compared to $9 \AA$ for minority-spin electrons). When we compare the numerically calculated attenuation length with that of the analytical solution of the three-dimensional equation (as derived in the Appendix B), a much better agreement is obtained. The results are the following: analytically $39 \AA$ versus nu- merically $38 \AA$ for majority-spin electrons, and for minorityspin electrons both the analytical and numerical calculations result in an attenuation length of $16 \AA$. The good agreement between the numerical calculations and the analytical formula is due to the fact that the angular distribution for the analytic solution for a ratio of $\lambda_{i} / \lambda_{e} \approx 5$ is fairly close to the angular distribution that appears in the numerical calculations.

In Fig. 8, we have changed both the $\mathrm{NiFe}$ and Co layer thicknesses, so that also the filtering properties of the Co layer improves as the layers become thicker. The MC in this situation increases exponentially with magnetic layer thickness, but it does not saturate. Note that there is a trade-off between MC and magnitude of the collector current, the latter goes down seriously as the layers thickness increases.

\section{CONCLUSIONS}

A model for the hot-electron transport in the spin-valve transistor has been presented. The transport in the base is described by the Boltzmann equation, in which elastic and inelastic scattering are taken into account in every individual layer of the transistor base. Elastic interface scattering is also included, but without spin dependence. The specific properties of the emitter and collector Schottky diodes are used as boundary conditions for the Boltzmann equation, and finally the equation is solved numerically. From the model we obtain the hot-electron attenuation and the evolution of the angular distribution of momentum throughout the SVT, from which the collector current and the magnetocurrent is calculated.

We demonstrated that the hot-electron attenuation depends on the local angular distribution of momentum, which by itself is related to previous (elastic) scattering events, and the emitter and collector properties. We show that broadening of the angular distribution due to elastic scattering at an interface leads to an increase of the effective bulk attenuation length of the layers after that interface. We also find that the magnetocurrent is changed by interface scattering even if it is taken to be independent of spin. A comparison between the full 3D calculation and a one-directional description shows that when elastic scattering is significant, the true attenuation lengths are markedly larger than those predicted in the one-directional model from the scattering parameters for elastic and inelastic scattering. If elastic scattering is important, the effective decay for a given set of scattering parameters can only be determined with a 3D calculation of the angular distribution through the device.

The calculations show that elastic scattering may be the primary reason for the small collector currents obtained in the SVT experimentally. A particularly dramatic reduction of the collector current was found to occur for strong elastic interface scattering. It thus appears that an increase in the interface quality could provide a significant increase in the transfer ratio. We also find that a high magnetocurrent of several $100 \%$ can be obtained for moderate thickness of the magnetic layers, due to the exponential thickness dependence of the current. 


\section{ACKNOWLEDGMENTS}

This research was supported by the Dutch Technology Foundation (STW) TEL 66.4025 and the European Community [MEL-ARI ESPIRIT 23.307 (SPIDER)] and [Brite Euram 13RPR-CT 96-0295 (HOTSEAMS)]. The research of R.J. was made possible by The Royal Netherlands Academy of Arts and Sciences (KNAW). Furthermore, the authors (R.V. and A.V.) thank the CEA for the generous hospitality.

\section{APPENDIX A: NUMERICAL PROCEDURE}

In this appendix the numerical procedure is presented, with which Eq. (7) is solved in a multilayer base with interface scattering, while also complying with the special boundary conditions due to the Schottky diodes.

Essential in this method is that we split Eq. (7) into two equations, one for electrons moving in the positive $x$ direction $\left[f^{>}(x, \mu)\right]$ and one for electrons moving in the negative $x$ direction $\left[f^{<}(x, \mu)\right]$ :

$$
\begin{aligned}
\mu \frac{\partial f^{>}(x, \mu)}{\partial x}= & -\left[\frac{1}{\lambda_{e}}+\frac{1}{\lambda_{i}}\right] f^{>}(x, \mu)+\frac{1}{2 \lambda_{e}} \\
& \times \int_{0}^{1} f^{>}(x, \mu) d \mu+S^{>}(x), \\
\mu \frac{\partial f^{<}(x, \mu)}{\partial x}= & -\left[\frac{1}{\lambda_{e}}+\frac{1}{\lambda_{i}}\right] f^{<}(x, \mu)+\frac{1}{2 \lambda_{e}} \\
& \times \int_{-1}^{0} f^{<}(x, \mu) d \mu+S^{<}(x) .
\end{aligned}
$$

Here, $S^{>}(x)$ and $S^{<}(x)$ are seen as backward source terms that represent inscattering of electrons that after an elastic scattering event move in the opposite direction (from moving to the left to moving to the right or vice versa). The $S^{>}(x)$ and $S^{<}(x)$ are defined as

$$
\begin{aligned}
& S^{>}(x)=\frac{1}{2 \lambda_{e}} \int_{-1}^{0} f^{<}(x, \mu) d \mu, \\
& S^{<}(x)=\frac{1}{2 \lambda_{e}} \int_{0}^{1} f^{>}(x, \mu) d \mu .
\end{aligned}
$$

In order to solve the set of coupled equations (A1)-(A4) for a single base layer of thickness $W$, we start with Eq. (A1) and solve it numerically for $x=0 \rightarrow W$ without taking into account $S^{>}(x)$, because it is unknown, but in every step calculating $S^{<}(x)$ and storing it into the computer memory. Next, we use Eq. (A2) to calculate $f^{<}(x, \mu)$ for $x=W \rightarrow 0$ while now using the previously stored values of $S^{<}(x)$ and calculating $S^{>}(x)$ at all positions. After the whole loop $(x$ $=0 \rightarrow W \rightarrow 0)$, the procedure is repeated until a selfconsistent solution is obtained.
In order to numerically solve Eq. (A1) we first discretize $\mu$ into $n$ directions $\left(\mu_{1, \ldots, n}\right)$, with $n=64$, so that $f^{>}(x, \mu)$ becomes $f_{i}^{>}(x)$. We can then convert the integral term in Eq. (A1) into a sum as

$$
\int_{0}^{1} f^{>}(x, \mu) d \mu \approx \sum_{i=1, \ldots, n} a_{i} f^{>}\left(x, \mu_{i}\right)=\sum_{i=1, \ldots, n} a_{i} f_{i}^{>}(x),
$$

in which $a_{i}$ are the coefficients for numerical integration. We have used a trapezoid like integration rule with optimally chosen $\mu$ values and $a_{1}=\mu_{1}+\left(\mu_{2}-\mu_{1}\right) / 2, \quad a_{i}=\left(\mu_{i+1}\right.$ $\left.-\mu_{i-1}\right) / 2$, and $a_{n}=\left(\mu_{n}-\mu_{n-1}\right) / 2$. Now Eq. (A1) can be written in a matrix form, in which the numerical integration coefficients are the elements of a matrix:

$$
\begin{gathered}
{\left[\begin{array}{c}
\mu_{1} \\
\mu_{2} \\
\vdots \\
\mu_{n}
\end{array}\right] \cdot \frac{\partial}{\partial x}\left[\begin{array}{c}
f_{1}^{>}(x) \\
f_{2}^{>}(x) \\
\vdots \\
f_{n}^{>}(x)
\end{array}\right]=-\left(\frac{1}{\lambda_{e}}+\frac{1}{\lambda_{i}}\right)\left[\begin{array}{c}
f_{1}^{>}(x) \\
f_{2}^{>}(x) \\
\vdots \\
f_{n}^{>}(x)
\end{array}\right]} \\
+\frac{1}{2 \lambda_{e}}\left[\begin{array}{cccc}
a_{1} & a_{2} & \cdots & a_{n} \\
a_{1} & a_{2} & \cdots & a_{n} \\
\vdots & & \ddots & \vdots \\
a_{1} & a_{2} & \cdots & a_{n}
\end{array}\right] \\
\times\left[\begin{array}{c}
f_{1}^{>}(x) \\
f_{2}^{>}(x) \\
\vdots \\
f_{n}^{>}(x)
\end{array}\right]+\left[\begin{array}{c}
S_{1}^{>}(x) \\
S_{2}^{>}(x) \\
\vdots \\
S_{n}^{>}(x)
\end{array}\right]
\end{gathered}
$$

or in a more compact notation

$$
\begin{aligned}
\frac{\partial}{\partial x}\left[\begin{array}{c}
f_{1}^{>}(x) \\
f_{2}^{>}(x) \\
\vdots \\
f_{n}^{>}(x)
\end{array}\right]=\left[\mu^{-1}\right]\left(\left[W_{\text {in }}\right]-\left[W_{\text {out }}\right]\right)\left[\begin{array}{c}
f_{1}^{>}(x) \\
f_{2}^{>}(x) \\
\vdots \\
f_{n}^{>}(x)
\end{array}\right] \\
\left.+\left[\begin{array}{c}
S_{1}^{>}(x) \\
S_{2}^{>}(x) \\
\vdots \\
S_{n}^{>}(x)
\end{array}\right]\right),
\end{aligned}
$$

in which we have introduced the in- and outscattering matrices $\left[W_{\text {in }}\right]$ and $\left[W_{\text {out }}\right]$, respectively, defined as $\left[W_{\text {in }}\right]$ $=\left(2 \lambda_{e}\right)^{-1}\left[M_{\text {int }}\right]$ and $\left[W_{\text {out }}\right]=\left(\lambda_{e}^{-1}+\lambda_{i}^{-1}\right)\left[M_{i d}\right]$, with the identity matrix $\left[M_{i d}\right]$ and the special integration matrix $\left[M_{\text {int }}\right]$ : 


$$
\left[M_{\text {int }}\right]=\left[\begin{array}{cccc}
a_{1} & a_{2} & \cdots & a_{n} \\
a_{1} & a_{2} & \cdots & a_{n} \\
\vdots & \vdots & \ddots & \vdots \\
a_{1} & a_{2} & \cdots & a_{n}
\end{array}\right] .
$$

The next step is to discretize $x$, so that the differential can be written as the difference of $f_{\mu}^{>}(x)$ and $f_{\mu}^{>}(x+d x)$, in which care should be taken that $d x$ is small enough to justify this procedure (in our case $d x=0.1 \AA$ ). The following iteration formula's then result:

$$
\begin{aligned}
& {\left[\begin{array}{c}
f_{1}^{>} \\
f_{2}^{>} \\
\vdots \\
f_{n}^{>}
\end{array}\right]_{x+d x}=\left[\begin{array}{c}
f_{1}^{>} \\
f_{2}^{>} \\
\vdots \\
f_{n}^{>}
\end{array}\right]_{x}+d x\left[\mu^{-1}\right]} \\
& \left.\times\left(\left[W_{\text {in }}\right]-\left[W_{\text {out }}\right]\right)\left[\begin{array}{c}
f_{1}^{>} \\
f_{2}^{>} \\
\vdots \\
f_{n}^{>}
\end{array}\right]_{x}+\left[\begin{array}{c}
S_{1}^{>} \\
S_{2}^{>} \\
\vdots \\
S_{n}^{>}
\end{array}\right]_{x}\right), \\
& {\left[\begin{array}{c}
S_{1}^{<} \\
S_{2}^{<} \\
\vdots \\
S_{n}^{<}
\end{array}\right]_{x+d x}=\left[W_{i n}\right]\left[\begin{array}{c}
f_{1}^{>} \\
f_{2}^{>} \\
\vdots \\
f_{n}^{>}
\end{array}\right]_{x}}
\end{aligned}
$$

and similarly for $f^{<}$and $S^{>}$, which are used in the calculations for $x=W \rightarrow 0$.

In a multilayer, the bulk scattering in the different layers can be easily implemented by calculating the $\left[W_{i n}\right]$ and $\left[W_{\text {out }}\right]$ matrices based on the local scattering parameters in the respective layers. But at the interface we have to connect the Boltzmann equations and introduce interface scattering. For the elastic interface scattering we use the parameter $D$ that represents the interface diffusivity. From the incident distribution of electrons, a fraction $(1-D)$ crosses the interface without being scattered, while a fraction $D$ will scatter elastically. The elastic interface scattering is assumed to be isotropic, such that a fraction $D / 2$ of the electrons will still move in the forward direction, and so this term can be written as

$$
\begin{aligned}
& {\left[\begin{array}{c}
f_{1}^{>} \\
f_{2}^{>} \\
\vdots \\
f_{n}^{>}
\end{array}\right]_{\text {layer } i+1}=(1-D)\left[M_{i d}\right]\left[\begin{array}{c}
f_{1}^{>} \\
f_{2}^{>} \\
\vdots \\
f_{n}^{>}
\end{array}\right]_{\text {layer } i}+\frac{D}{2}\left[M_{\text {int }}\right] } \\
& \times\left[\begin{array}{c}
f_{1}^{>} \\
f_{2}^{>} \\
\vdots \\
f_{n}^{>}
\end{array}\right]_{\text {layer } i}+\left[\begin{array}{c}
S_{1}^{>} \\
S_{2}^{>} \\
\vdots \\
S_{n}^{>}
\end{array}\right]_{\text {layer } i} \cdot(\mathrm{A} 11)
\end{aligned}
$$

But the other $D / 2$ will be scattered in the backward direction, so that we have to incorporate this as a backward source at the interface, which can be written as

$$
\left[\begin{array}{c}
S_{1}^{<} \\
S_{2}^{<} \\
\vdots \\
S_{n}^{<}
\end{array}\right]_{\text {layer } i}=\frac{D}{2}\left[M_{\text {int }}\right]\left[\begin{array}{c}
f_{1}^{>} \\
f_{2}^{>} \\
\vdots \\
f_{n}^{>}
\end{array}\right]_{\text {layer } i},
$$

and again similarly for $f^{<}$and $S^{>}$, which are used in the calculations for $x=W \rightarrow 0$.

\section{APPENDIX B: ANALYTICAL SOLUTION}

In this appendix an analytical solution of Eq. (7) is derived. As known, the Boltzmann equation will roughly attenuate exponentially with position $x$ inside the metallic base, so we make the following ansatz:

$$
f(x, \mu)=A(\mu) e^{-x / \lambda_{e f f}} .
$$

When this is substituted into Eq. (7) and we divide by the common $e^{-x / \lambda_{\text {eff }}}$ factor, it can be written after some rearrangements as

$$
A(\mu)=\frac{1}{2 \lambda_{e}} \frac{\int_{-1}^{1} A(\mu) d \mu}{\left(\frac{1}{\lambda_{e}}+\frac{1}{\lambda_{i}}-\frac{\mu}{\lambda_{e f f}}\right)} .
$$

Next, by integrating both sides of Eq. (B2) over $\mu$ from -1 to 1 and by dividing the common integral out, one obtains

$$
1=\frac{\lambda_{e f f}}{2 \lambda_{e}} \ln \left[\frac{\left(\lambda_{e}+\lambda_{i}\right) \lambda_{e f f}+\lambda_{e} \lambda_{i}}{\left(\lambda_{e}+\lambda_{i}\right) \lambda_{e f f}-\lambda_{e} \lambda_{i}}\right] .
$$

From this equation the value of $\lambda_{e f f}$ is easily evaluated, given the values of $\lambda_{e}$ and $\lambda_{i}$. In Fig. 3, the different combinations of $\lambda_{e}$ and $\lambda_{i}$ resulting in a particular value of $\lambda_{\text {eff }}$ are shown. The angular distribution $A(\mu)$ for this $\lambda_{e f f}$ can be written in normalized (at $\mu=1$ ) form as

$$
A^{N}(\mu)=\frac{\left(\frac{1}{\lambda_{e}}+\frac{1}{\lambda_{i}}-\frac{1}{\lambda_{e f f}}\right)}{\left(\frac{1}{\lambda_{e}}+\frac{1}{\lambda_{i}}-\frac{\mu}{\lambda_{e f f}}\right)} .
$$

Although the above solution is only valid for a certain type of angular distribution for given values of $\lambda_{e}$ and $\lambda_{i}$, it gives a direct relation between the elastic and inelastic scattering lengths and the resulting attenuation length $\lambda_{e f f}$. Unfortunately, only numerical methods can be applied to solve Eq. (7) in the case of the spin-valve transistor, because special boundary conditions (due to the Schottky barriers), different scattering lengths (due to the multilayer in the base), and interfacial scattering have to be taken into account. 
${ }^{1}$ D. J. Monsma, J. C. Lodder, Th. J. A. Popma, and B. Dieny, Phys. Rev. Lett. 74, 5260 (1995).

${ }^{2}$ D. J. Monsma, R. Vlutters, and J. C. Lodder, Science 281, 407 (1998).

${ }^{3}$ P. S. Anil Kumar, R. Jansen, O. M. J. van't Erve, R. Vlutters, P. de Haan, and J. C. Lodder, J. Magn. Magn. Mater. 214, L1 (2000).

${ }^{4}$ R. Jansen, P. S. Anil Kumar, O. M. J. van't Erve, R. Vlutters, P. de Haan, and J. C. Lodder, Phys. Rev. Lett. 85, 3277 (2000).

${ }^{5}$ T. Yamauchi and K. Mizushima, Phys. Rev. B 61, 8242 (2000).

${ }^{6}$ T. Yamauchi and K. Mizushima, Phys. Rev. B 58, 1934 (1998).

${ }^{7}$ S.M. Sze, Physics of Semiconductor Devices, 1st ed. (Wiley, New York, 1969).

${ }^{8}$ S. M. Sze, C. R. Crowell, G. P. Carey, and E. E. LaBate, J. Appl. Phys. 37, 2690 (1966).

${ }^{9}$ J. J. Quinn, Phys. Rev. 126, 1453 (1962).

${ }^{10}$ E. Zarate, P. Apell, and P. M. Echenique, Phys. Rev. B 60, 2326 (1999).

${ }^{11}$ R. E. Camley and J. Barnas, Phys. Rev. Lett. 63, 664 (1989).

${ }^{12}$ J. Barnas, A. Fuss, R. E. Camley, P. Grunberg, and W. Zinn, Phys. Rev. B 42, 8110 (1990).

${ }^{13}$ T. Valet and A. Fert, Phys. Rev. B 48, 7099 (1993).
${ }^{14}$ S. Zhang and P. M. Levy, J. Appl. Phys. 69, 4786 (1991).

${ }^{15}$ N. W. Ashcroft and N. D. Mermin, Solid State Physics (Saunders College, Philadelphia, 1976).

${ }^{16}$ S. M. Sze, Physics of Semiconductor Devices, 2nd ed. (Wiley, New York, 1981).

${ }^{17}$ D. K. Guthrie, L. E. Harrell, G. N. Henderson, P. N. First, T. K. Gaylord, E. N. Glytsis, and R. E. Leibenguth, Phys. Rev. B 54, 16972 (1996).

${ }^{18}$ R. Knoren, K. H. Bennemann, R. Burgermeister, and M. Aeschlimann, Phys. Rev. B 61, 9427 (2000).

${ }^{19}$ W. H. Rippard and R. A. Buhrman, Phys. Rev. Lett. 84, 971 (2000).

${ }^{20}$ M. Aeschlimann, M. Bauer, S. Pawlik, W. Weber, R. Burgermeister, D. Oberli, and H. C. Siegmann, Phys. Rev. Lett. 79, 5158 (1997).

${ }^{21}$ M. K. Weilmeier, W. H. Rippard, and R. A. Buhrman, Phys. Rev. B 59, R2521 (1999).

${ }^{22}$ L. D. Bell, Phys. Rev. Lett. 77, 3893 (1996).

${ }^{23}$ P. Niedermann, L. Quattropani, K. Solt, I. MaggioAprile, and O. Fischer, Phys. Rev. B 48, 8833 (1993). 